\title{
Local tuning of fused silica thermal expansion coefficient using femtosecond laser
}

\author{
Pieter Vlugter, ${ }^{*}$ Erica Block, and Yves Bellouard \\ Galatea Lab, STI/IMT, Ecole Polytechnique Fédérale de Lausanne (EPFL), 2002 Neuchâtel, Switzerland
}

(Received 15 January 2019; revised manuscript received 9 April 2019; published 10 May 2019)

\begin{abstract}
Femtosecond laser exposure of fused silica in the nonablative regime can lead to various localized bulk modifications of the material structure. In this paper, we show that these laser-induced modifications can be used to tune silica thermal expansion properties permanently. In particular, we demonstrate that a given exposer regime leads to lower thermal expansion than the bulk, while other exposure conditions yield the opposite results. This remarkable property enables the possibility to engineer a given thermal expansion behavior by selectively exposing a material volume to a femtosecond laser beam. This finding opens up opportunities for a variety of integrated precision instruments and optical devices for which inertness to thermal fluctuations is essential.
\end{abstract}

DOI: 10.1103/PhysRevMaterials.3.053802

\section{INTRODUCTION}

Thermal expansion of material is one of the most challenging engineering problems for precision instruments, in particular for devices seeking high-dimensional stability over a broad range of temperatures. Starting from the discovery of Invar, a great deal of effort has been put into finding materials approaching zero-thermal expansion while having other interesting physical properties, such as optical transparency or low weight. This has led to the development of specialized ceramics, such as Zerodur, which achieves record low thermal expansion, approaching $10^{-9} \mathrm{~K}^{-1}$, two orders of magnitude smaller than fused silica, which has the lowest coefficient for thermal expansion (CTE) among "single materials," and two to three orders less than Invar. The low CTE in Zerodur is achieved by adding two constituents in a composite, with one constituent exhibiting a negative CTE balancing the positive CTE of the other [1]. Further efforts have been made in the search of negative CTE materials in complex material systems [2,3]. Another approach to tune macroscopic material properties is based on metamaterials, where a repeating microscale unit cell has a programmable CTE and dictates the effective CTE of the metamaterial at the macroscale. This microscale unit cell consists of a trusslike architecture, made out of different materials having different positive CTE [4,5]. Recent progress in additive $3 \mathrm{D}$ polymerization techniques has enabled the implementation of composite microstructures [6,7], demonstrating theoretical models for tuning and optimizing thermal expansion behaviors. These concepts of tailoring material properties will undoubtedly broaden the material selection space for low- to zero-CTE materials. In this paper, we propose an approach based on femtosecond laser exposure to engineer locally the CTE of bulk fused silica, according arbitrary shapes, and without adding any material to the raw substrate.

The method consists of exposing the substrate in a nonablative regime with a laser beam consisting of pulses of

\footnotetext{
*pieter.vlugter@epfl.ch
}

tens to hundreds of femtoseconds. The high-peak power achieved at the focal spot triggers nonlinear absorption events, leading to structural changes in the matter. In fused silica, three different regimes of structural modifications have been described [8]. In the sequel, we focus our attention to the first two regimes, found for lower energies. Just above the modification threshold, a regime (so-called regime I) is found for low pulse energy $(<\sim 200 \mathrm{~nJ})$, and pulses shorter than $200 \mathrm{fs}$. These structural modifications are characterized by an apparent homogeneously modified volume, in which a higher refractive index is observed [9]. The change in refractive index is explained by a local densification of the material $[10,11]$. The change in volume has been confirmed using a direct method, based on micromechanics [12]. Increasing further the pulse energy under similar pulse durations yields another type of modification (so-called type II), characterized by the occurrence of self-organized, periodic structures spaced by about half the laser wavelength [13], and commonly referred to as nanogratings [14]. These periodic arrangement of nanoplanes exhibit form-birefringence [15] that indicates a fine modulation of the refractive index [13]. Later, it was shown experimentally that these nanoplanes consist of a porous structure [16]. Using SAXS analysis, the porosity was further investigated and two pore diameters $(10$ and $30 \mathrm{~nm})$ were found inside these planes [17]. This regime is associated with a net localized volume expansion [18].

In addition, it has been shown that the modified material may show significant changes in refractive index [9], mechanical properties such as Young's modulus [11,19], and chemical etching susceptibility [20]. The change in volume induces anisotropic stress in the surrounding bulk material [21]. These property changes allow for embedding different types of functionalities in the same substrate, such as, for instance, integrated optics [22-25], optical networks with fluidic [26,27] and/or mechanical systems [28].

The structural change observed for the two types of modifications, density and Young's modulus, gives a priori evidence that the thermal expansion of the exposed volumes is likely to be altered after laser exposure. Here, we demonstrate that we can tune the thermal-expansion coefficient of fused silica 


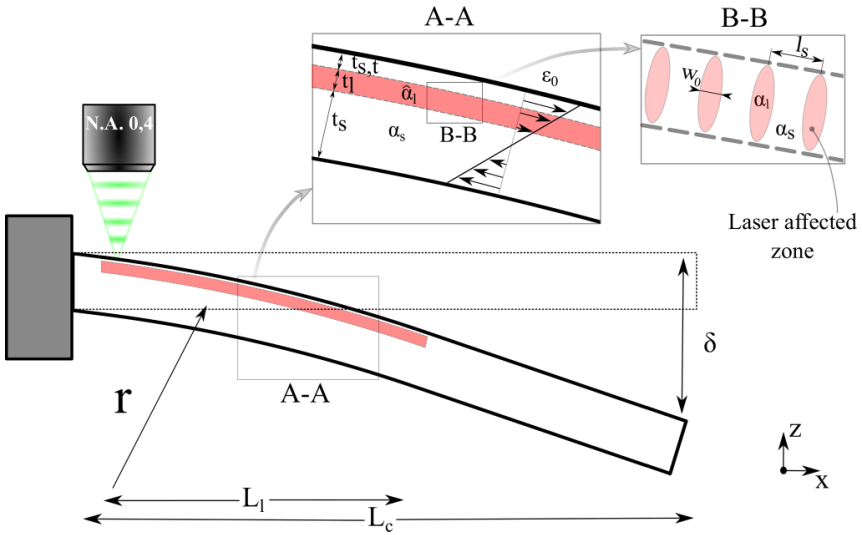

FIG. 1. A schematic cross section of a cantilever where the near top layer is exposed by a femtosecond laser. The red zone represents the laser-affected zones (LAZs). The cantilever bends with the radius $r$, and $\delta$ is the amplified motion of the cantilevers tip. In the magnified image, A-A is the initial strain $\left(\epsilon_{0}\right)$ depicted and the thickness of the different layers labeled. In the magnified image, B-B are the single written lines depicted as red ellipses. The line spacing, $l_{s}$, and modified width, $w_{0}$, are defined on the figure. Furthermore, the thicknesses of the different layers are denoted as $t_{s, t}, t_{l}$, and $t_{s}$, and the length of the cantilever is $L_{c}$, and the modified zone length is $L_{l}$. Finally, $\alpha_{s}, \alpha_{l}$, and $\hat{\alpha}_{l}$ are the coefficients of thermal expansion of the pristine material, the modified material, and of the modified layer (which is a composition of laser-affected and -unaffected material), respectively.

and engineer it, further expanding the application potential of femtosecond laser structuring of glass.

\section{WORKING PRINCIPLE AND MODEL}

Fused silica exhibits one of the lowest CTEs among commercially available materials $\left(\sim 0.6 \cdot 10^{-6} \mathrm{~K}^{-1}\right)$, only surpassed by a few others, such as Zerodur and Sitall. This already low CTE for the bulk substrate material makes thermal expansion measurement of laser-induced modifications difficult to measure due to the very small amplitude of the effects. To overcome this problem, we use a method based on a cantilever amplifying mechanism illustrated in Fig. 1. The cantilever is locally exposed just below its top surface [12,18], such that exposed and unexposed regions form an equivalent bimorph structure made of two materials, with different CTEs, stacked one onto another. The laser-exposed layer consists of an ensemble of lines forming a plane parallel to the cantilever surface (Fig. 1, B-B). Just like a thermal bimorph, a difference of thermal expansion coefficients between pristine and laser-affected zones will cause the structure to bend upon temperature changes, upward or downward, depending on the sign of the difference between the two CTEs. The cantilever s tip displacement $(\delta)$ results from an amplification effect due to the strain introduced inside laser-affected zones (LAZ). The relation between $\delta$, exposed length, and radius of curvature is given in Eqs. (1). There, $L_{l}$ denotes the cross-sectional length of the LAZ, $L_{c}$ the length of the cantilever, and $\mathrm{r}$ is the radius of curvature as shown in Fig. 1. In our model, we are representing cross sections of laser-affected lines by

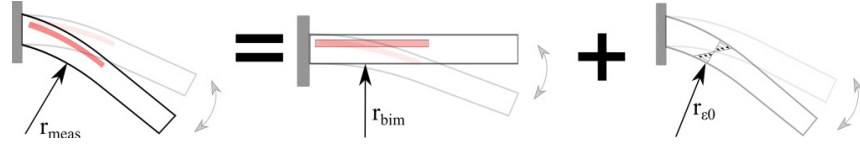

FIG. 2. Schematic illustrating the superposition of two effects at the origin of the bending of the cantilever due to temperature variations: the thermal bending due to the CTE's difference and the bending effect due to the initial strain in the material.

plain ellipsoids that define our representative volume elements (RVEs). LAZs may have finer structures-in particular, for the so-called nanogratings regime where one can find nanoplanes, themselves consisting in nanopores. However, for this particular problem, and as we will see later on, the level of granularity for describing the laser-affected volume is sufficient for describing the thermoelastic behavior of the different regimes at the scale we are considering.

$$
\begin{aligned}
\delta(T) & =\frac{L_{l}\left(L_{c}-L_{l} / 2\right)}{r(T)}, \\
\text { let } \quad L_{l} & =\eta L_{c} \quad \text { with } \eta \in[0,1], \\
\delta & =\left[\frac{L_{c}^{2}}{r}\right]\left(\eta-\frac{\eta^{2}}{2}\right) .
\end{aligned}
$$

Timoshenko [29] and others [30-32] formulated an analytical description of the thermal deflections of a bimorph/multilayer structure; however, these expressions are not sufficient to describe our problem, as in these models it is commonly assumed a nonprestressed state as initial condition. In our previous work [12], we have shown that the modified regions shrink or expand when the exposure conditions correspond to regimes I and II, respectively. Consequently, these volume variations induce an initial bending, prestressing the cantilever and causing a linear variation of the bending strain $\left(\epsilon_{0}\right)$ along the $z$ axis. The magnitude of the strain induced during initial laser exposure depends, in particular, on the Young's modulus of the bulk, unexposed material. Since the Young's modulus is itself temperature dependent $[33,34]$ the strain induced in the pristine material as a result of the laser exposure of the top layer also becomes temperature dependent. (Note that Bell et al. [35] used this effect to compensate for the positive CTE of fused silica.) Therefore, the bending of the cantilever resulting from a temperature variation contains two terms: one that is inherent to the preloading effect due to the laser exposure, and another one that truly depends on the difference of CTE between laser-affected zones and pristine ones. This situation is depicted in Fig. 2. To estimate the preponderance of these two effects, a mathematical model is derived based on a method inspired from Ref. [36] in which a closed form solution for multilayer systems is described. The final result is shown in Eq. (2). In this model, we introduce an external moment that causes the initial bending radius. Furthermore, as a first approximation, we assume that the Young's modulus is linearly depending on temperature $[33,34]$. Using a Taylor expansion, we then separate the thermal bending into one term, $r_{\text {bim }}(T)$, that accounts for the difference in CTEs, and another one, $r_{\epsilon_{0}}(T)$, that accounts for the effect of the initial cantilever stress loading. This separation is possible with the 
assumption that deformation due to the exposure is much larger than the ones from the thermal expansion $\left(1 / r_{\text {meas }} \ll\right.$ $\left.1 / r_{0}\right)$. As we will see later on in the section discussing the experimental results, this approximation is justified. Further simplifications are made by stating that the thickness of the laser-affected zone is much smaller compared to the thickness of the unaffected ones (i.e., $t_{l} \ll t_{s}$ ). The derivation of the analytical expression shown in Eq. (2) is further detailed in the Appendix.

The simplified, analytical model for the measured cantilever bending caused by a temperature variation writes

$$
\begin{aligned}
\frac{1}{r_{\text {meas }}} & =\frac{1}{r_{\text {bim }}}+\frac{1}{r_{\epsilon_{0}}} \\
& =\left[6\left(\hat{\alpha}_{l, 0}-\alpha_{s, 0}\right)\left(\frac{\hat{E}_{l, 0}}{E_{s, 0}}\right)\left(\frac{t_{l}}{t_{s}^{2}}\right)-\frac{1}{E_{s, 0}}\left(\frac{d E_{s}}{d T}\right) \frac{1}{r_{0}}\right] \Delta T .
\end{aligned}
$$

Where $\alpha$ is the CTE, $E$ is the Young's modulus, $t$ the thickness of the different layers. Subscripts $\cdot_{S}$ and ${ }_{l}$ refer to the pristine and modified (laser-affected zone) volumes, respectively. The additional ${ }_{, 0}$ subscript means that we consider the parameter at room temperature.

In practice, the laser-exposed layer is inhomogeneous and is composed of a set of parallel lines, with a characteristic spacing, as depicted in Fig. 1. The exposed layer therefore blends unmodified and modified material. As a consequence, the material constants $\hat{E}_{l, 0}$ and $\hat{\alpha}_{l, 0}$ have to be expressed in terms of exposed and unexposed volumes using the rule of mixture, as shown in Eqs. (3) and (4). Where $V_{l}$ and $V_{s}$ are the volume fractions of laser-modified and unmodified volumes, respectively, and expressed in Eqs. (5) and (6). $l_{s}$ is the line spacing between the centers of subsequent modified volumes and $w_{0}$ is the width of the modified volume, specified in Fig. 1 (cross section B-B). In this problem, the material property constants, $\alpha_{l, 0}$ and $E_{l, 0}$, are unknown. For the Young's modulus, $E_{l, 0}$, we assumed its values from previous work [11,19]:

$$
\begin{gathered}
\hat{E}_{l, 0}=\frac{E_{l, 0} E_{s, 0}}{V_{l} E_{s, 0}+V_{s} E_{l, 0}}, \\
\hat{\alpha}_{l, 0}=\alpha_{l, 0} V_{l}+\alpha_{s, 0} V_{s}, \\
V_{s}=\frac{l_{s}-w_{0}}{l_{s}}, \\
V_{l}=1-V_{s} .
\end{gathered}
$$

\section{A. Experimental procedure}

Eight cantilevers were fabricated out of $500-\mu \mathrm{m}$-thick fused silica substrate (Corning 7980-0F), using a two-step process combining femtosecond laser exposure and chemical etching as described in Ref. [18]. The substrates used have a 1000-ppm OH content and traces of $\mathrm{Cl}$ in the order of 100 ppm (class-III fused silica). To create the bimorph structure, the cantilevers are exposed after etching near the top surface, as depicted in Fig. 1. To study a wide range of exposure conditions, two femtosecond laser systems, having different pulse characteristics, are used. The first one emits 50 fs-pulse (an OPA from Amplitude Systèmes, emitting at $850 \mathrm{~nm}$ )

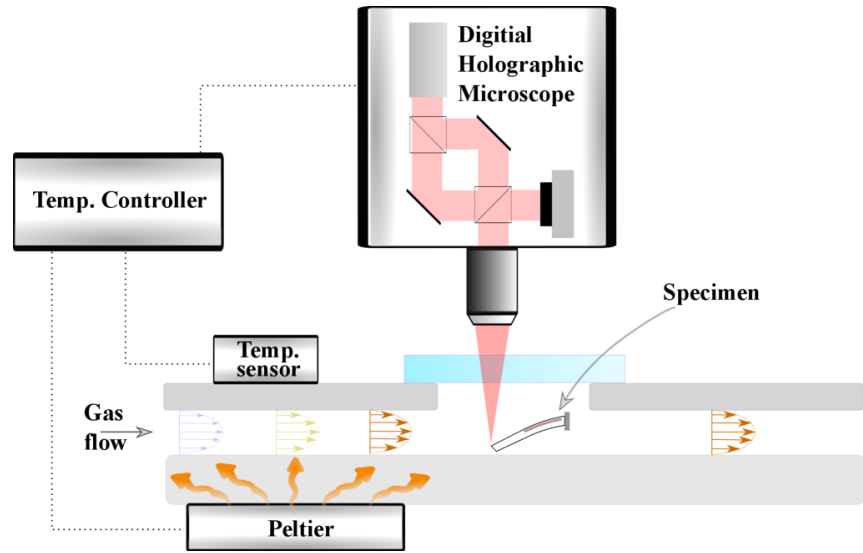

FIG. 3. Schematic of the experimental setup for measuring cantilever deflections. The exposed cantilever is placed in a "miniature" environmental chamber and thermalized, thanks to a laminar flow of a preheated/precooled inert gas $\left(\mathrm{N}_{2}\right)$. The deflection is measured using a digital holographic microscope (DHM) that measures the motion of the cantilever surface through a window.

and is capable of producing regime-I-type transformations, while the second one is an Yb-doped fiber amplified system (Amplitude Systèmes, $380 \mathrm{fs}$ at a wavelength of $1030 \mathrm{~nm}$ ) and is capable of producing regime-II-type transformations.

To investigate the thermal-dependent cantilever deflection, a stable and uniform temperature distribution is needed, which is particularly challenging considering the low thermal conductivity of fused silica. To achieve this goal, a dedicated environmental chamber was realized, in Fig. 3 a schematic is depicted of the chamber - part of the experimental setup. The low thermal conductivity precludes the use of a conduction mechanism through the specimen to achieve an homogeneous temperature. Furthermore, to be able to probe optically the out-of-plane motion of the bimorph structure, an optical viewport is needed on top of the chamber. This window represents a thermal leak in our chamber, as it is not well isolated, which may create a temperature gradient in the volume where the specimen is placed. To overcome these two issues, we choose an approach based on gas convection. In practice, a preheated (or precooled) inert gas $\left(\mathrm{N}_{2}\right)$ flows in a laminar regime across the chamber volume and thermalizes the specimen by convection. A close-loop control algorithm is used to keep the gas at the desired temperature and to apply controlled-temperature heating or cooling ramps. Heating and cooling of the gas is done in elongated $1 \mathrm{~m}$-long serpentine channels made in an aluminum substrate cooled or heated up using thermoelectric devices. With this scheme, the measured thermal fluctuations throughout the chamber volume are in the range of $\sim 10 \mathrm{mK}$. A digital holographic microscope (DHM) is then used to measure the cantilever out of plane deflections, with a noise-floor below $+/-8 \mathrm{~nm}$. The temperature controller (that regulates the temperature in the chamber) and the DHM are synchronized, so recorded holograms are acquired at known temperature points. A commercial software (Koala from Lyncée Tec) reconstructs an intensity and phase image from the recorded hologram, where the intensity image is used, in a postprocessing step, to track parasitic motions of 
the sample in lateral directions inherently induced by the chambers thermal expansion itself. The phase image is used to measure out-of-plane motions of the cantilever tip relative to a reference taken on the specimen. To prevent further parasitic thermal stress, the specimen is placed on a kinematic mount supported by precision ruby spheres ensuring three well-defined contact points. With this strategy, the specimen can expand freely.

\section{RESULTS}

Two types of experiments are conducted: one where the thermal response is studied for different laser exposure conditions, and another one, for which laser-exposure conditions are kept constant, but the filling factor of the exposed volume is varied. In this second set of experiments, the filling factor was tested by changing the line spacing between the individual lines $\left(l_{s}\right.$ in Fig. 1). The motivation is to validate our model [Eq. (2)] by effectively changing the average CTE $\left(\hat{\alpha}_{l, 0}\right)$ of the LAZ and the initial bending strain $\left(\epsilon_{0}\right)$.

\section{A. Regime II and the effect of annealing}

The modifications were written using the fiber laser emitting $250 \mathrm{~nJ}$-pulses at a translation speed of $1.7 \mathrm{~mm} / \mathrm{s}$ and a repetition rate of $800 \mathrm{kHz}$. Further, we investigated the effect of annealing this sample. Indeed, it is known that the femtosecond laser-exposed regions exhibit an increase in defects, such as nonbridging oxygen hole center (NBOHC) and E centers [37]. Witcher et al. [38] investigated the NBOHC removal as function of a wide range of thermal annealing temperatures. They showed with Raman measurements and fluorescent spectroscopy that the NBOHC defects completely disappear with an annealing temperature of $300{ }^{\circ} \mathrm{C}$, whereas the laser-modified $\mathrm{Si}-\mathrm{O}$ ring structures remains. The latter may be suppressed at an annealing temperature of $900{ }^{\circ} \mathrm{C}$. Furthermore, Zhang et al. [39] found that the intensity of the D2 peak remains quasiconstant, with annealing temperatures below $500{ }^{\circ} \mathrm{C}$. In our experiment, we adopted the annealing strategy discussed in Ref. [38] to remove the NBOHC to minimize possible long-term changes; for instance, due to the outgassing of trapped oxygen or quenching of defects. The thermal response of the samples is measured, before and after annealing, using the previously described experimental setup. The annealing step is done in a furnace under normal atmospheric conditions, at $300{ }^{\circ} \mathrm{C}$ for 10 hours. Additionally, the initial deflection at room temperature, due to the volume expansion after femtosecond laser exposure, is measured using a white light interferometer (WYKO NT1100) and is reported in Table I-these values are used to estimate $r_{0}$. It should be pointed out that annealing has no large effect on $\delta_{0}$ and, therefore, variation of the $r_{\epsilon_{0}}(T)$ is small upon annealing.

The graph in Fig. 4, considers an example of thermal tip displacements in nanometers as function of temperature. The slope of the curve before annealing is negative. In other words, the cantilever bends down due to a temperature increase. After annealing, an opposite effect is observed. One might conclude that the difference of the thermal expansions $\left(\Delta \alpha=\alpha_{s}-\alpha_{l}\right)$ is switching signs due to the annealing step. This behavior
TABLE I. Measured initial tip deflections $\left(\delta_{0}\right)$ after exposure and annealing with measurement uncertainties within $200 \mathrm{~nm}$. The third column lists the difference between the two measurements.

\begin{tabular}{lccc}
\hline \hline $\begin{array}{c}\delta_{0}(\mu \mathrm{m}) \\
l_{s}(\mu \mathrm{m})\end{array}$ & $\begin{array}{c}\delta_{0}(\mu \mathrm{m}) \\
\text { Before annealing }\end{array}$ & $\begin{array}{l}\text { after annealing } \\
\text { Difference }(\%)\end{array}$ \\
\hline 2 & -76.1 & -78.7 & 3.4 \\
6 & -65.7 & -64.7 & -1.5 \\
8 & -50.5 & -50.2 & -0.6 \\
10 & -40.8 & -40.8 & 0 \\
\hline \hline
\end{tabular}

is confirmed when observing the thermal displacement rate $(\partial \delta / \partial T)$ for all the line spacing, see top graph in Fig. 5.

However, when subtracting the term related to the prestress effect $\left(\delta_{\epsilon_{0}}\right)$ (see previous paragraph) from the measurement, we observe that the thermal bending rate only caused by the thermal expansion difference $\left(\delta_{\text {bim }}\right)$ is negative for both cases (see the bottom graph in Fig. 5). From the latter, it becomes apparent that the magnitude of the thermal strain rate decreases when annealing is applied, hence $\alpha_{l}$ converges to $\alpha_{s}$, which may indicate that some fine reorganizations of the material matrix might be taking place. From the bottom graphs that exclude the initial strain, we conclude that in regime II, the modified volume expands more than the unmodified region, hence $\alpha_{l}>\alpha_{s}$. Indeed the magnitude of $\delta_{\text {bim }}$ decreases as function of the line spacing, as the exposed volume decreases.

\section{B. Thermal response vs deposited energy regimes I and II}

In this section, we discuss the thermal deflections as function of the exposure conditions and, in particular, the pulse width and deposited energy. The regime-I modifications are created by irradiating the specimen with 50 fs-pulse length

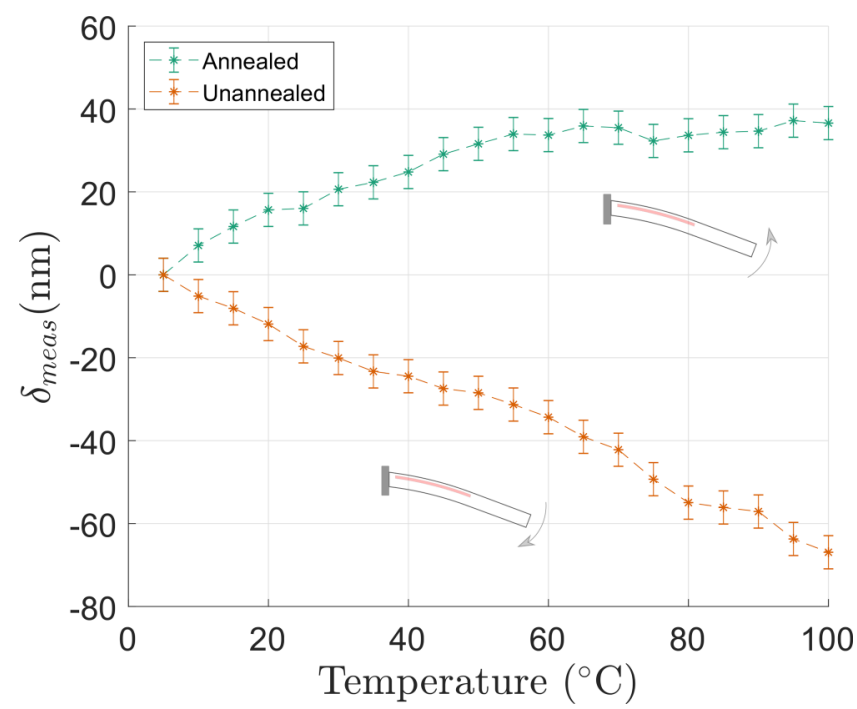

FIG. 4. The measured thermal deflection $\left(\delta_{\text {meas }}\right)$ for annealed and unannealed specimens in the case of regime-II exposure. Exposure conditions: $E_{p}=250 \mathrm{~nJ}, E_{d}=10 \mathrm{~J} / \mathrm{mm}^{2}$, pol $=90^{\circ}$, and $l_{s}=4 \mu \mathrm{m}$. 


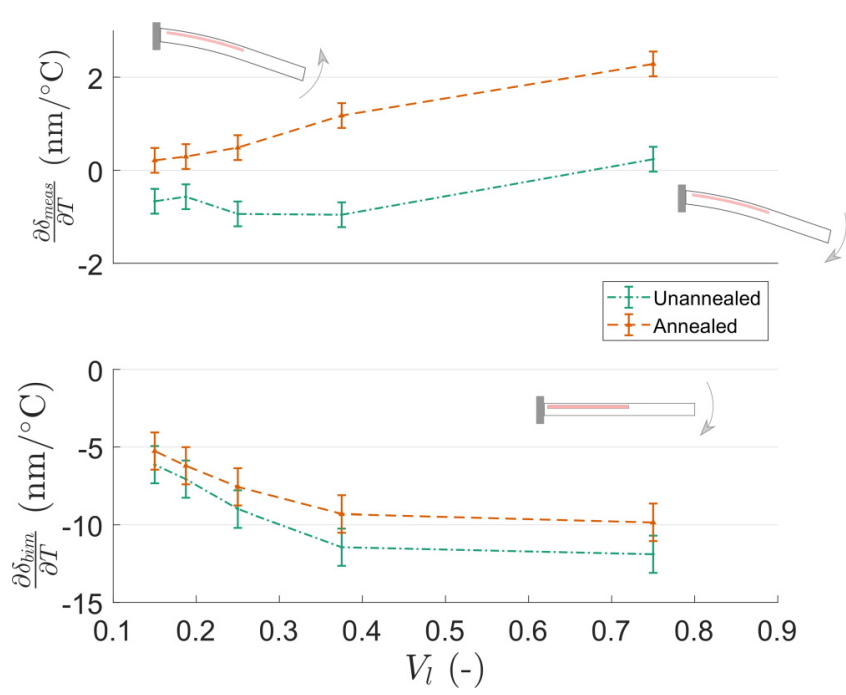

FIG. 5. The thermal deflection rate $(\partial \delta / \partial T)$ for the annealed and unannealed case as function of the volume fraction of exposed material $\left(V_{l}\right)$ in the case of regime-II exposure. The top figure depicts the measured rate and the bottom figure is the thermal deflection rate only due to the CTE differences $\left(\partial \delta_{\text {bim }} / \partial T\right)$. Exposure conditions: $E_{p}=250 \mathrm{~nJ}, E_{d}=10 \mathrm{~J} / \mathrm{mm}^{2}, \mathrm{pol}=90^{\circ}$.

and $250 \mathrm{~nJ}$ pulse energy, while regime II is obtained using $230 \mathrm{~nJ}$ pulses using the longer pulse laser. The scanning speed is adjusted to reach a desired deposited energy level. The number of pulses is directly expressed by the ratio between repetition rate and scanning speed. In regime I, it ranges between 26.5 and 1600 per micron and for regime II, between 18 and 1200 per micron. The line spacing was fixed and set to $4 \mu \mathrm{m}$, hence more than two times the width of the laser-affected zone. This choice is motivated by observations of cross-talk phenomena [11] when the spacing becomes comparable or smaller than the laser-affected width. In addition, for regime II, two polarizations are chosen, $0^{\circ}$ and $90^{\circ}$ (defined as the angle between the electrostatic vector field and the writing direction), to validate the effect of the self-organized nanograting orientations. Figure 6 depicts the thermal deflection rate $\left(\partial \delta_{\text {bim }} / \partial T\right)$, only resulting from the bimorph effect. The thermal rate of the cantilevers exposed with type-I modification is positive and hence, an undeformed bimorph structure would bend upward opposite to what is observed for regime-II-modified cantilevers as discussed in the previous paragraph. This observation further suggests that the CTE of a type-I modification is smaller than the one of the pristine material. The deflection $s$ magnitude in a type-I regime increases strongly with an increasing deposited energy and nearly saturates at fluences above $200 \mathrm{~J} / \mathrm{mm}^{2}$. The type-II modification shows a slight increase in absolute thermal deflection for low deposited energies, while above fluences of $20 \mathrm{~J} / \mathrm{mm}^{2}$, a decrease is observed. A similar trend (peak, followed by a decrease) has been observed for etching rates versus deposited energy experiments [40] as well as for volume variation and deposited energy range and, finally, the stress versus deposited energy, suggesting a correlation between the two [18]. In Ref. [18], we suggested that the abrupt decreasing volume variation was due to crack formation in the modified volume. The same reasoning could explain the

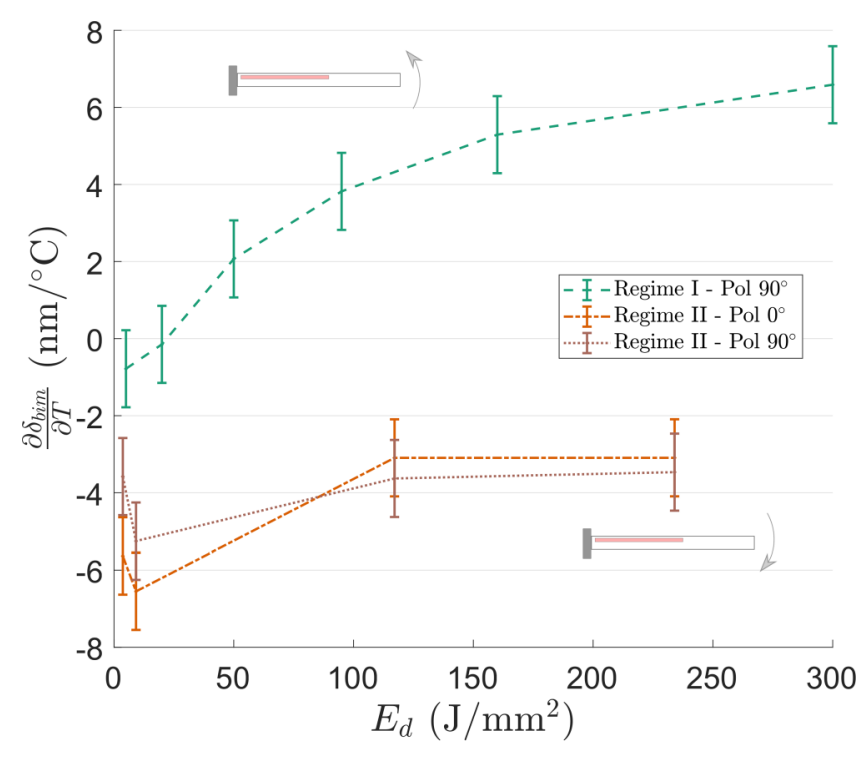

FIG. 6. The thermal deflection rate due to the CTE differences $\left(\partial \delta_{\text {bim }} / \partial T\right)$ of a cantilever with regime-I and -II modifications, as function of deposited energy. The pulse energy is for regime I $250 \mathrm{~nJ}$ and for regime II is $230 \mathrm{~nJ}$; the latter is done for a polarization of $0^{\circ}$ and $90^{\circ}$. The animation indicates the bending direction.

decrease in thermal expansion for higher deposited energy: the induced cracks regions not being able to transmit stress into the surrounding volume, which will lower the effective thermal expansion of the complete volume.

\section{ESTIMATION OF THE CTE OF LASER-EXPOSED SILICA}

Let us consider Eqs. (2) to (6) and rewrite them as depicted in Eq. (7). It shows that $\alpha_{l, 0}$ has one term inversely proportional to $E_{l, 0}$. To estimate the CTE, it is therefore necessary to make an appropriate assumption of the Young's modulus as the two variables are coupled:

$$
\alpha_{l, 0}=\frac{1}{r_{\text {bim }}} \frac{1}{6} \frac{t_{s}^{2}}{t_{l}}\left(\frac{V_{s}}{V_{l}}+\frac{E_{s, 0}}{E_{l, 0}}\right) \frac{1}{\Delta T}+\alpha_{s, 0} .
$$

The Young's modulus was indirectly measured in Ref. [19] for regime II and directly for regime I in Ref. [11], for similar modified structures as in this paper. In regime II, there is evidence in the literature that the Young's modulus would decrease along with an increasing deposited energy, which is assumed to be correlated to an increase of porosity. First, a qualitative experimental study was made to determine the degree of porosity of a type-II modification using SAXS analysis [17] and it showed that the porosity increases significantly with an increasing number of pulses. Second, Athanasiou et al. [19] correlated experimental obtained values with the degree of porosity by an empirical model given for a type-II modification.

Unfortunately, we do not have specific data of Young's modulus versus porosity in the case of laser-exposed specimens. Numerous empirical relationships between the degree of porosity and Young's modulus are reported [41] for porous silica or, for instance, for silica gel and low- $k$ films, and are 


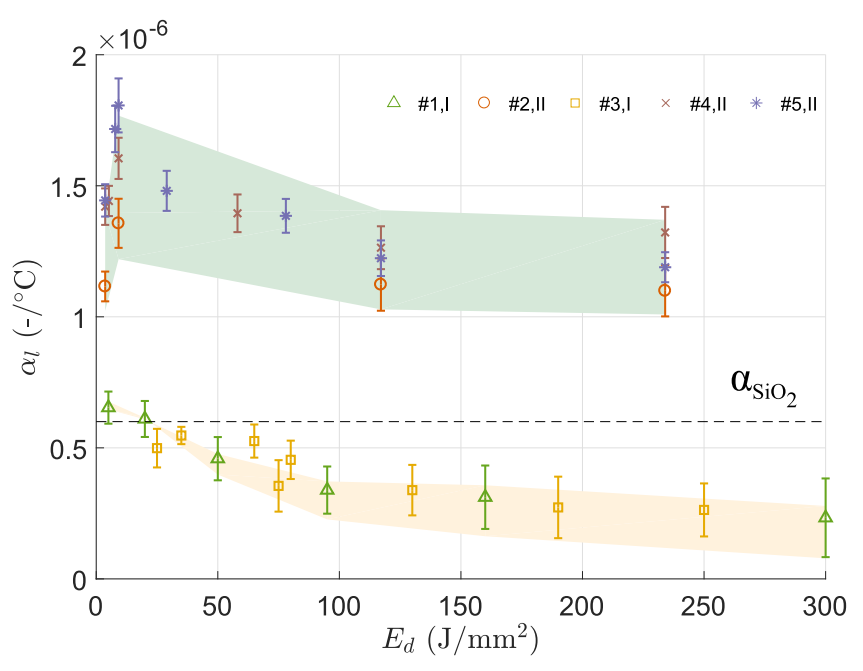

FIG. 7. The estimated CTE for regimes I and II as function of deposited energy, with both a polarization of $90^{\circ}$. Numbers 1 and 2 are the results corresponding to the substrates discussed in the previous section, No. 3 , No. 4 , and No. 5 are the results corresponding to additional substrates. The shaded area depicts the uncertainty on this estimation based on the uncertainty of the Young's modulus. The value for pristine silica is shown as a reference.

summarized in Ref. [42]. The reported experimental data, corresponding to a $50 \%$ porosity, range from 2 to $20 \mathrm{GPa}$, depending on the processing methods. It illustrates how strong the impact of the degree of porosity is and the degree of uncertainty concerning these estimates. Nevertheless, we use these data to estimate the CTE for a range of plausible estimated Young's modulus values. Here, we take the mean values for both regimes reported in Refs. [11,19] that correspond to similar exposure conditions, i.e., for regime I, $76.2 \mathrm{GPa}$, and regime II (with a polarization of $90^{\circ}$ ) $30 \mathrm{GPa}$, and estimate the CTE with a Young's modulus uncertainty of $+/-50 \%$, which is quite conservative. Even with such high uncertainty, the CTE fluctuates within a small range of values. The results [obtained using Eqs. (7)] are shown in Fig. 7. Numbers 1 and 2 are the results corresponding to the substrates discussed in the previous section. To test the repeatability of the CTE tuning process, three additional substrates (No. 3, No. 4, and No. 5) were exposed and measured. The results are also shown in Fig. 7. Labels I and II indicate the modification regime. The graphs (shown with dashed lines) represent the mean values, with error bars based on estimates of the geometrical measurement errors for laser-affected zones (extracted from Ref. [11]), on the instrument errors (white light interferometer and DHM) as well as error estimates on material parameters based on previous works $[19,34]$. The boundaries of the shaded areas around the graphs illustrate the CTE approximation for the upper and lower boundary uncertainty on the Young's modulus. The estimated CTE for regime I converges to a value a factor of 2 lower, roughly, than the pristine material. It approaches $0.3 \mathrm{ppm} /{ }^{\circ} \mathrm{C}$ for deposited energies above $100 \mathrm{~J} / \mathrm{mm}^{2}$. The CTE's mean value for regime II makes a sharp increase for deposited energies below $20 \mathrm{~J} / \mathrm{mm}^{2}$ and drops for higher values; however, this trend is rather insignificant due to the high uncertainty on the Young's modulus. Nevertheless, we can state that the CTE increases strongly, approximately by a factor of 2 .

\section{CONCLUSION}

In this paper, it has been established that the CTE of fused silica is modified by femtosecond laser irradiation in a notable manner, i.e., typically in the order of factor of 2 for the laser exposure conditions investigated. More interestingly, we have shown that the sign of CTE change depends on the type of laser-induced modifications that is governed by pulse duration and/or the pulse energy. While densified zones (so-called type I) display a decrease of CTE, nanogratings (type II) show the opposite trend, with respect to the pristine material. A parametric study to determine the most relevant exposure conditions has been conducted, and shows clearly an evolution of the thermal expansion behavior.

This is a first step toward selective tuning of the thermal expansion behavior of microdevices by constructing a modulated CTE landscape in 3D, controlled by laser and writing patterns. This paper contributes to highly thermally stable optical components, such as, for instance, substrates containing complex wave-guide networks [22-24], like MachZehnder interferometers [43], resonators [44], laser cavities, or recently demonstrated photonic lanterns [25] and quantum information processing circuits [45]. It also goes beyond integrated optics, as silica is also used as a material for high-precision mechanical components such as flexures [11], such as, for instance, mirror suspensions used in gravitational wave detectors [35]. This paper also contributes to expand the use of silica for high-precision components in the watch industry and, in particular, using it for the fabrication of reliable thermo-compensated mechanical oscillators.

This research offers a paradigm shift for thermally compensated optics and opens research horizons for exploring further femtosecond laser tailoring of functional material properties in three dimensions and, in particular, thermal expansion properties, pushing the limits of 3D integration.

\section{ACKNOWLEDGMENTS}

The Galatea Lab is thankful to the sponsorship of Richemont. The authors are thankful to Amplitude Systèmes for providing the experimental 50-fs OPA laser source used in this study and for the LMTS laboratory of EPFL for providing the DHM.

In this paper, P.V. wrote the draft paper, performed the measurements including the testing chamber design, interpreted the measurement results, and implemented the analytical model. E.B. set up the 50-fs laser that was used in this experiment. Y.B. designed and supervised the research and revised the paper. All the authors discussed the results.

\section{APPENDIX: DERIVATION OF THE ANALYTICAL MODEL}

We derive the analytical expression based on a method described in Ref. [36] and adapted to our problem. We define 
the strain of a three-layer cantilever as

$$
\epsilon(z)=c+\frac{z-t_{n}}{r}+\frac{z-t_{n}}{r_{0}} \text { for }-t_{s} \leqslant z \leqslant t_{l}+t_{s, t} .
$$

$c$ is the constant strain component, $r$ and $r_{0}$ is the bending radius due to thermal deflections and the initial volume expansion, respectively. $t_{n}$ is the distance between the neutral line and the bottom surface. $z$ is the position variable depicted in Fig. 1 and is defined as zero at the interface of the bottom and exposed layers. The domain of the strain $[\epsilon(z)]$ is defined as the sum of the thicknesses of the different layers, $t_{s}, t_{l}$, and $t_{s, t}$, which are defined in Fig. 1. The stresses $\left(\sigma_{s}, \sigma_{l}\right.$, and $\left.\sigma_{s, t}\right)$ in the different layers are expressed by the set of Eqs. (A2)-(A4):

$$
\begin{gathered}
\sigma_{s}=E_{s}(T)\left(\epsilon-\alpha_{s}\right) \Delta T \quad \text { for } ;-t_{s} \leqslant z \leqslant 0, \\
\sigma_{l}=E_{l}(T)\left(\epsilon-\alpha_{l}\right) \Delta T \quad \text { for } 0 \leqslant z \leqslant t_{l}, \\
\sigma_{s, t}=E_{s}(T)\left(\epsilon-\alpha_{s}\right) \Delta T \quad \text { for } t_{l} \leqslant z \leqslant t_{l}+t_{s, t} .
\end{gathered}
$$

$E$. and $\alpha$. are the Young's modulus and thermal expansion, respectively. The subscripts $l_{l}$ and ${ }_{s}$ refer to the modified (laser-affected zone) and pristine volumes, respectively. In this problem, we consider that the Young's modulus is temperature dependent and is expressed using a first-order linearized form [33]:

$$
\begin{aligned}
& E_{s}(T)=E_{s, 0}+\frac{d E_{s}}{d T} \Delta T, \\
& E_{l}(T)=E_{l, 0}+\frac{d E_{l}}{d T} \Delta T .
\end{aligned}
$$

A solution for radius, $r$, can be found by solving the three following equations: Eq. (A7), the sum of forces due to the uniform strain component should be zero, Eq. (A8), the sum of forces due to the bending strain should be zero, Eq. (A9), and, last, the sum of moments should be equal to an external moment. The external moment represents the moment induced by the initial volume expansion of the modified material:

$$
\begin{gathered}
E_{s}\left(c-\alpha_{s} \Delta T\right) t_{s}+E_{l}\left(c-\alpha_{l} \Delta T\right) t_{l}+E_{s}\left(c-\alpha_{s} \Delta T\right) t_{l}=0, \\
\int_{-t_{s}}^{0} \frac{E_{s}\left(z-t_{n}\right)}{r} d z+\int_{0}^{t_{l}} \frac{E_{l}\left(z-t_{n}\right)}{r} d z+\int_{t_{l}}^{t_{l}+t_{s, t}} \frac{E_{l}\left(z-t_{n}\right)}{r} d z=0, \\
\int_{-t_{s}}^{0} \sigma_{s}\left(z-t_{n}\right) d z+\int_{0}^{t_{l}} \sigma_{l}\left(z-t_{n}\right) d z+\int_{t_{l}}^{t_{l}+t_{s, t}} \sigma_{s, t}\left(z-t_{n}\right) d z=M .
\end{gathered}
$$

In Eq. (A9), the moment $M$ represents the moment which causes the initial bending induced by volume expansion of the LAZ. Substituting and rewriting the latter equation to the bending radius:

$$
\frac{1}{r}+\frac{1}{r_{0}}=\frac{3\left\{E_{s}\left(c-\alpha_{s}\right)\left[t_{s}^{2}-t_{s, t}\left(2 t_{l}+t_{s, t}\right)\right]-E_{l} t_{l}^{2}\left(c-\alpha_{l}\right)\right\} \Delta T+M}{E_{s, 0}(C 1+C 3)+E_{l, 0} C 2} .
$$

With $C_{1}, C_{2}$, and $C_{3}$ as geometrical constants:

$$
\begin{gathered}
C_{1}=t_{s}^{2}\left(2 t_{s}+3 t_{n}\right), \\
C_{2}=t_{l}^{2}\left(2 t_{l}-3 t_{n}\right), \\
C_{3}=t_{s, t}\left[6 t_{l}^{2}+6 t_{l} t_{s, t}+t_{t, s t}^{2}-3 t_{n}\left(2 t_{l}+t_{s, t}\right)\right] .
\end{gathered}
$$

Linearizing Eq. (A10) using Taylor expansion around $\Delta T=0$ gives

$$
\begin{aligned}
\frac{1}{\hat{r}}+\frac{1}{r_{0}}= & \frac{M}{E_{s, 0}\left(C_{1}+C_{3}\right)+E_{l, 0} C_{2}}-\frac{\frac{d E_{s}}{d T}\left(C_{1}+C_{3}\right)+\frac{d E_{l}}{d T} C_{2}}{\left(E_{s, 0}\left(C_{1}+C_{3}\right)+E_{l, 0} C_{2}\right)^{2}} M \Delta T \\
& +\frac{3\left\{E_{s, 0}\left(c-\alpha_{s}\right)\left[t_{s}^{2}-t_{s, t}\left(2 t_{l}+t_{s, t}\right)\right]-E_{l, 0} t_{l}^{2}\left(c-\alpha_{l}\right)\right\}}{E_{s, 0}\left(C_{1}+C_{3}\right)+E_{l, 0} C_{2}} \Delta T .
\end{aligned}
$$

Notice that the material constants are not dependent on temperature anymore. Using this linearized equation, we can express the moment $M$ in terms of $r_{0}$ by setting $\Delta T=0$ :

$$
\frac{1}{r_{0}}=\frac{M}{E_{s, 0}\left(C_{1}+C_{3}\right)+E_{l, 0} C_{2}} .
$$

Substituting $M$ into Eq. (A14) and subtracting the constant term gives the thermal dependant radius $\hat{r}$ :

$$
\frac{1}{\hat{r}}=-\frac{\frac{d E_{s}}{d T}\left(C_{1}+C_{3}\right)+\frac{d E_{l}}{d T} C_{2}}{E_{s, 0}\left(C_{1}+C_{3}\right)+E_{l, 0} C_{2}} \frac{1}{r_{0}} \Delta T+\frac{3\left\{E_{s, 0}\left(c-\alpha_{s}\right)\left[t_{s}^{2}-t_{s, t}\left(2 t_{l}+t_{s, t}\right)\right]-E_{l, 0} t_{l}^{2}\left(c-\alpha_{l}\right)\right\}}{E_{s, 0}\left(C_{1}+C_{3}\right)+E_{l, 0} C_{2}} \Delta T .
$$


Considering the dimensions of the exposed cantilever, we can state that $C_{1}+C_{3} \gg C_{2}$ so Eq. (A16) becomes

$$
\frac{1}{\hat{r}}=-\frac{d E_{s}}{d T} \frac{1}{E_{s, 0}} \frac{1}{r_{0}} \Delta T+\frac{3\left\{E_{s, 0}\left(c-\alpha_{s}\right)\left[t_{s}^{2}-t_{s, t}\left(2 t_{l}+t_{s, t}\right)\right]-E_{l, 0} t_{l}^{2}\left(c-\alpha_{l}\right)\right\}}{E_{s, 0}\left(C_{1}+C_{3}\right)} \Delta T .
$$

Further simplification, based on order of magnitude, would lead to

$$
\frac{1}{\hat{r}}=\frac{6\left(E_{l, 0} t_{l}\left(\alpha_{l}-\alpha_{s}\right)\right)}{E_{s, 0} t_{s}^{2}} \Delta T-\frac{d E_{s}}{d T} \frac{1}{E_{s, 0}} \frac{1}{r_{0}} \Delta T .
$$

In this equation, we found the terms for thermal deflection due to the thermal expansion difference and for prestrained material directly related to the temperature-dependent Young's modulus. Indeed, this linearized equation holds only for small deflections around the equilibrium position at $\Delta T=0$, hence for $\frac{1}{\hat{r}} \ll \frac{1}{r_{0}}$.

[1] R. B. Roberts, R. J. Tainsh, and G. K. White, Cryogenics 22, 566 (1982).

[2] K. Takenaka, Sci. Technol. Adv. Mater. 13, 013001 (2012).

[3] H. Yamamoto, T. Imai, Y. Sakai, and M. Azuma, Angew. Chem. Int. Ed. 57, 8170 (2018).

[4] O. Sigmund and S. Torquato, J. Mech. Phys. Solids 45, 1037 (1997).

[5] R. Lakes, Appl. Phys. Lett. 90, 221905 (2007).

[6] Q. Wang, J. A. Jackson, Q. Ge, J. B. Hopkins, C. M. Spadaccini, and N. X. Fang, Phys. Rev. Lett. 117, 175901 (2016).

[7] J. Qu, M. Kadic, A. Naber, and M. Wegener, Sci. Rep. 7, 40643 (2017).

[8] C. Hnatovsky, R. S. Taylor, P. P. Rajeev, E. Simova, V. R. Bhardwaj, D. M. Rayner, and P. B. Corkum, Appl. Phys. Lett. 87, 014104 (2005).

[9] K. M. Davis, K. Miura, N. Sugimoto, and K. Hirao, Opt. Lett. 21, 1729 (1996).

[10] J. W. Chan, T. Huser, S. Risbud, and D. M. Krol, Opt. Lett. 26, 1726 (2001).

[11] Y. Bellouard, T. Colomb, C. Depeursinge, M. Dugan, A. A. Said, and P. Bado, Opt. Express 14, 8360 (2006).

[12] Y. Bellouard, A. Champion, B. McMillen, S. Mukherjee, R. R. Thomson, C. Pépin, P. Gillet, and Y. Cheng, Optica 3, 1285 (2016).

[13] Y. Shimotsuma, P. G. Kazansky, J. Qiu, and K. Hirao, Phys. Rev. Lett. 91, 247405 (2003).

[14] W. Yang, E. Bricchi, P. G. Kazansky, J. Bovatsek, and A. Y. Arai, Opt. Express 14, 10117 (2006).

[15] E. Bricchi, B. G. Klappauf, and P. G. Kazansky, Opt. Lett. 29, 119 (2004).

[16] M. Lancry, B. Poumellec, J. Canning, K. Cook, J.-C. Poulin, and F. Brisset, Laser Photon. Rev. 7, 953 (2013).

[17] S. Richter, A. Plech, M. Steinert, M. Heinrich, S. Döring, F. Zimmermann, U. Peschel, E. B. Kley, A. Tünnermann, and S. Nolte, Laser Photon. Rev. 6, 787 (2012).

[18] A. Champion and Y. Bellouard, Opt. Mater. Express 2, 789 (2012).

[19] C.-E. Athanasiou and Y. Bellouard, Micromachines 6, 1365 (2015).

[20] A. Marcinkevičius, S. Juodkazis, M. Watanabe, M. Miwa, S. Matsuo, H. Misawa, and J. Nishii, Opt. Lett. 26, 277 (2001).

[21] B. McMillen and Y. Bellouard, Opt. Express 23, 86 (2015).
[22] Y. Sikorski, A. A. Said, P. Bado, R. Maynard, C. Florea, and K. A. Winick, Electron. Lett. 36, 226 (2000).

[23] K. Minoshima, A. M. Kowalevicz, I. Hartl, E. P. Ippen, and J. G. Fujimoto, Opt. Lett. 26, 1516 (2001).

[24] G. D. Marshall, M. Ams, and M. J. Withford, Opt. Lett. 31, 2690 (2006).

[25] R. R. Thomson, T. A. Birks, S. G. Leon-Saval, A. K. Kar, and J. Bland-Hawthorn, Opt. Express 19, 5698 (2011).

[26] R. M. Vazquez, R. Osellame, D. Nolli, C. Dongre, H. van den Vlekkert, R. Ramponi, M. Pollnau, and G. Cerullo, Lab. Chip 9, 91 (2009).

[27] Y. Bellouard, A. Said, M. Dugan, and P. Bado, Mater. Res. Soc. Symp. 782, 63 (2003).

[28] Y. Bellouard, A. Said, and P. Bado, Opt. Express 13, 6635 (2005).

[29] S. Timoshenko, J. Opt. Soc. Am. 11, 233 (1925).

[30] M. Benabdi and A. A. Roche, J. Adhes. Sci. Technol. 11, 281 (1997).

[31] W. C. Young and R. G. Budynas, Roark's Formulas for Stress and Strain, 7th ed. (McGraw-Hill, New York, USA, 2002).

[32] M. Christophersen, B. Shapiro, and E. Smela, Sens. Actuators B Chem. 115, 596 (2006).

[33] S. Spinner, J. Am. Ceram. Soc. 39, 113 (1956).

[34] Y. Bao and G. Chen, Meas. Sci. Technol. 27, 065101 (2016).

[35] C. J. Bell, S. Reid, J. Faller, G. D. Hammond, J. Hough, I. W. Martin, S. Rowan, and K. V. Tokmakov, Class. Quantum Gravity 31, 065010 (2014).

[36] C.-H. Hsueh, Appl. Phys. 91, 9652 (2002).

[37] H.-B. Sun, S. Juodkazis, M. Watanabe, S. Matsuo, H. Misawa, and J. Nishii, J. Phys. Chem. B 104, 3450 (2000).

[38] J. J. Witcher, W. J. Reichman, L. B. Fletcher, N. W. Troy, and D. M. Krol, Opt. Mater. Express 3, 502 (2013).

[39] F. Zhang, Y. Yu, C. Cheng, Y. Dai, H. Zhang, and J. Qiu, Appl. Phys. B 117, 53 (2014).

[40] S. Rajesh and Y. Bellouard, Opt. Express 18, 21490 (2010).

[41] T. Adachi and S. Sakka, J. Mater. Sci. 25, 4732 (1990).

[42] J. M. Rimsza and J. Du, J. Am. Ceram. Soc. 97, 772 (2014).

[43] G. Li, K. A. Winick, A. A. Said, M. Dugan, and P. Bado, Opt. Lett. 31, 739 (2006).

[44] K. J. Vahala, Nature 424, 839 (2003).

[45] L. Sansoni, F. Sciarrino, G. Vallone, P. Mataloni, A. Crespi, R. Ramponi, and R. Osellame, Phys. Rev. Lett. 105, 200503 (2010). 